Cahiers de recherches médiévales

\title{
Étienne de Laigue et le commentaire de Jules César à la Renaissance
}

Marie-Élisabeth Boutroue

\section{(2) OpenEdition \\ Journals}

Édition électronique

URL : https://journals.openedition.org/crm/2571

DOI : $10.4000 / \mathrm{crm} .2571$

ISSN : 1955-2424

Éditeur

Honoré Champion

Édition imprimée

Date de publication : 30 juin 2007

Pagination : 159-172

ISSN : $1272-9752$

Référence électronique

Marie-Élisabeth Boutroue, "Étienne de Laigue et le commentaire de Jules César à la Renaissance », Cahiers de recherches médiévales [En ligne], 14 spécial | 2007, mis en ligne le 30 juin 2010, consulté le 15 décembre 2022. URL : http://journals.openedition.org/crm/2571 ; DOI : https://doi.org/10.4000/ crm.2571 


\section{酷M}

\section{Étienne de Laigue et le commentaire de Jules César à la Renaissance}

C'est à Paris que fut imprimé pour la première fois en français l'ensemble des textes qui constitue le corpus césarien. Des éditions latines du corpus avaient certes été produites auparavant: l'édition aldine de 1513, par exemple, avait connu un large succès un peu partout en Europe. Toutefois, du corpus césarien en français, n'était disponible que la traduction de la Guerre des Gaules par Robert Gaguin'. La Guerre civile, les œuvres qui constituent le pseudo-César, n'étaient quant à elles disponibles qu'en latin. Cette première publication en langue vernaculaire du corpus complet de César est en soi suffisamment importante pour qu'on lui porte quelque intérêt. Elle n'a cependant pas fait l'objet d'étude détaillée et l'on peut voir les pages qui suivent comme une tentative de réparation de ce qui est sans doute une injustice.

Le responsable de cette publication est un diplomate français, connu essentiellement par une activité intense au service de François $\mathrm{I}^{\mathrm{er}}$. Étienne de Laigue, seigneur de Beauvais en Berry, comme il aime à se désigner lui-même, a eu une carrière militaire sans doute brillante, suivie d'une activité de négociateur caractérisée par son extrême discrétion. Sans entrer dans l'exposé détaillé des diverses missions dont le chargea le roi de France $^{2}$, il suffit ici de rappeler ses plus brillants faits d'armes et les étapes essentielles de son activité diplomatique ${ }^{3}$. La préface du commentaire de Pline l'ancien, qu'il publia en 1530, fait allusion aux désordres de la guerre, dont le commentateur avoue être récemment sorti ${ }^{4}$. Lorsqu'on est militaire et diplomate dans ces années-là, le désordre de la guerre ne peut être lié qu'aux toutes récentes campagnes de François $\mathrm{I}^{\mathrm{er}}$ en Italie. Dès lors, il faut admettre qu'Étienne de Laigue était aux côtés de son souverain à Pavie et, sans doute, qu'il a pu bénéficier d'un otium obligatoire ensuite, aux côtés du souverain durant sa captivité ou, plus vraisemblablement, auprès des enfants de France à partir de 1526 : le dépouillement de sources archivistiques espagnoles permettrait peut-être de préciser cet aspect de sa carrière. Du côté français, je n'ai pas trouvé de preuve

\footnotetext{
${ }^{1}$ Sur la tradition de la traduction du De Bello gallico par Robert Gaguin, nous renvoyons à l'article de Frédéric Duval, «Le Livre des commentaires Cesar sur le fait des batailles de Gaule par Robert Gaguin (1485) ou de l'art de la transposition », CRM, n 13 spécial, année 2006, p. 167-182.

${ }^{2}$ Nous préparons une édition de certaines de ses œuvres et renvoyons ainsi à la publication en préparation.

${ }^{3}$ Le personnage lui-même est assez peu connu. Les mentions assez nombreuses dans les armoriaux concernent sa famille plus que notre diplomate lui-même. On se reportera à : Louis de Laigue, "Un soldat diplomate au XVI ${ }^{\mathrm{e}}$ siècle : ambassade extraordinaire de Beauvoys à Venise en 1536 », Revue d'histoire diplomatique, 1908 ; Marie-Elisabeth Boutroue, «Étienne de Laigue: un diplomate de la Renaissance, lecteur de Pline l'Ancien », Nouvelle revue du seizième siècle, $1992, \mathrm{n}^{\circ} 10$, p. 33-49.

${ }^{4}$ Étienne de Laigue, In omnes C. Plinii Secundi Naturalis historia argutissimi scriptoris libros, Stephani Aqucei,... commentaria, Paris, Galiot du Pré, 1530.
}

Cahiers de Recherches Médiévales, 14spé, 2007 
absolue de sa présence dans la péninsule ibérique à cette période; je dispose en revanche de quelques indices pour étayer ce qui n'est qu'une hypothèse.

Le premier de ces indices est contenu dans l'extrait du privilège du roi qui ouvre la traduction française du corpus césarien, dans la première édition de 1531: le texte mentionne, ce qui est absolument courant, l'intention du roi de France de promouvoir ou restaurer les belles lettres; la phrase qui suit ce que je suis tentée de voir comme un topos de la littérature des extraits de privilège s'appuie cependant sur un second motif, moins banal celui-là : «Parquoy nous ces choses considerees, ... Aussy les bons services que ledict de beauvoys nous a faictz et esperons quil nous fera au temps advenir. A iceluy avons permis et permettons de grace especiale par ces presentes quil puisse faire imprimer et vendre par lesdictz le Preux et Du Pré, lesdictz commentaires de la guerre civile par luy traduictz».

Il fallait d'ailleurs que ces services fussent d'importance - c'est le deuxième indice de ma recherche - pour qu'ils justifiassent en outre l'octroi au seigneur de Beauvais en Berry de l'ordre de Saint-Michel, réservé sous le règne de François I ${ }^{\text {er }}$ comme sous celui de ses prédécesseurs à des hommes nobles, ayant rendu de grands services à l'état ${ }^{5}$.

Les années qui suivent 1530 prouvent à l'évidence que la confiance du roi était bien placée. Les comptes de François I ${ }^{\mathrm{er}}$, la correspondance d'Eustace Chapuys, les nonciatures, mettent en évidence le rôle joué par Étienne de Laigue dans le dispositif diplomatique mis en œuvre par le roi. Dès 1531, justement, on le voit assez régulièrement stipendié par la Couronne pour des missions explicitement identifiées comme secrètes par le compte du roi. J'ai tenté de restituer, sous une forme largement abrégée quelques unes de ses missions diplomatiques entre 1531 et 1538 , date de sa mort.

Le 17 mai 1531, Étienne de Laigue est mentionné dans les comptes de François $\mathrm{I}^{\mathrm{er}}$. Il reçoit 200 écus soleil sur les deniers des parties casuelles. Le texte ne précise pas pour quelle mission l'ambassadeur est ainsi stipendié. Dix jours plus tard, il reçoit encore 1200 livres à titre d'avance sur les frais de voyage entrepris en Ecosse. La durée prévue de son voyage est de quatre mois. Le 8 novembre 1532, c'est une mission en Ecosse, qualifiée de secrète par les comptes de François I ${ }^{\text {er }}$ qui justifie la réception par le seigneur de Beauvais d'une somme de 400 écus soleil. En mars 1533, Étienne de Laigue reçoit encore 1000 livres tournois pour une mission dont le roi l'a chargé en Écosse. Un an plus tard, le 20 août 1534, Gervais Wain et Étienne de Laigue sont envoyés comme ambassadeurs auprès du Landgrave de Hesse. Le 22 août, mandement est fait au trésorier de l'Épargne de leur verser 920 livres pour ce voyage en Allemagne. Étienne de Laigue recevra encore 720 livres pour un voyage de quatre vingt-dix jours en Allemagne, comme ambassadeur du roi, en janvier 1535 .

En 1536, C'est sans doute encore notre homme qui est cité dans une lettre d'Ambrogio Ricalcati à Girolamo Verallo. Datée du 31 janvier 1536, écrite à Rome, elle mentionne l'arrivée à Amboise du trésorier de Beoves, porteur de lettres du secrétaire d'état datées du 23 avril.

${ }^{5}$ Pour l'inventaire des chevaliers del'ordre de Saint-Michel, on se reportera à Jean-François d'Hozier, Recueil historique des chevaliers de l'Ordre de Saint-Michel, vol. I, 1469-1560, publ. par Michel Popoff, Paris, le Léopard d'or, 1998 
Ce bref tableau des missions diplomatiques d'Étienne de Laigue n'est pas complet. Il suffit cependant à prouver l'intensité de son activité diplomatique dans les plus hautes affaires de l'état. Les missions en Écosse et en Angleterre sont particulièrement bien connues. Elles sont directement en rapport avec la volonté marquée de François $\mathrm{I}^{\mathrm{er}}$ de servir d'intermédiaire entre le roi d'Écosse, Jacques V, et le roi d'Angleterre Henry VIII que de nombreux conflits opposaient. En Allemagne, c'est la politique religieuse du roi de France qui inquiète et justifie l'envoi de deux ambassadeurs en la personne d'Étienne de Laigue et Gervais Wain ${ }^{6}$. Outre ces missions, on le retrouve encore en Italie, aux côtés de Georges de Selve, ambassadeur ordinaire près la Sérénissime, à un moment où il est de nouveau question de l'affaire du Milanais. En 1535-1536, l'un de ces derniers voyages le voit passer par la Suisse. Il s'agit alors de lever des troupes, pour préparer l'invasion de la Provence par les troupes impériales. Pour des raisons personnelles ou pour des raisons militaires, Étienne de Laigue devait rester ensuite en Avignon, où l'on apprend qu'il meurt en $1538^{7}$.

Une carrière aussi occupée pourrait sembler a priori peu favorable à la publication d'une œuvre d'érudition. Pourtant, entre 1530 et 1531 , notre ambassadeur ne porte pas moins de quatre œuvres à publication. Ces œuvres sont bien connues: ce sont d'abord deux œuvres qui ressortissent au genre du commentaire entendu au sens large, sur Pline d'une part, sur César d'autre part. Puis on trouve deux très courts opuscules respectivement intitulés Singulier traicté contenant la proprieté des Tortues, Escargotz, Genoilles et Artichauts et un éloge paradoxal intitulé Encomium brassicarum siue caulium, qui n'est peut-être qu'à demi paradoxal : certes le chou n'était pas un bien grand sujet pour un éloge, mais ses vertus thérapeutiques et nutritives étaient connues depuis l'Antiquité. Le commentaire sur l'Histoire Naturelle de Pline mentionne, en outre, un commentaire sur Martianus Capella, sans doute perdu.

Pour conclure, Étienne de Laigue ne semble pas avoir été un professionnel des belles lettres. Qu'il ait été éduqué à la mode humaniste ne fait aucun doute et, quelle que soit la part d'ombre qui plane sur sa biographie, son origine berrichonne, le fait que plusieurs membres de sa famille sont explicitement liés à Alciat, tout contribue à le rattacher assez solidement à cette lignée de gentilhommes cultivés dont la contribution à la paix ou à la guerre entre les nations n'avait pas pour nécessaire contrepartie une inculture de mauvais aloi.

Il faut à présent en venir à la traduction de César. On disposait naturellement d'éditions latines depuis le $\mathrm{XV}^{\mathrm{e}}$ siècle, pour les œuvres authentiquement césariennes, autant que pour le corpus du pseudo-César. On disposait également d'éditions donnant le corpus en entier et, parmi les plus remarquables de ces éditions, il convient de faire une place particulière à l'édition publiée par Alde Manuce en $1513^{8}$. Selon toute probabilité, Étienne de Laigue a eu cette édition entre

\footnotetext{
${ }^{6}$ Sur les missions de Gervais Wain et Étienne de Laigue, je renvoie à V. L. Bourrilly, Jacques Colin, abbé de Saint-Ambroise, Paris, 1905, p. 74-75.

${ }^{7}$ C'est une note de Claude Cottereau, en marge d'un manuscrit des Ogdoades de Guillaume du Bellay qui nous l'apprend. v. Paris, BnF, ms. lat. 5976, fol. 7

${ }^{8}$ Antoine Auguste Renouard, Annales de l'imprimerie des Alde ou histoire des trois Manuce et de leurs éditions, $3^{\mathrm{e}}$ édition, New Castle, Delaware, Oak Knoll Books, 1991, p. 60. On
} 
les mains ou l'une de celles qui en dérivaient dans les années suivantes. En Français, seule la Guerre des Gaules était disponible, dans la traduction de Robert Gaguin. Les autres textes, et tout particulièrement le De Bello ciuili n'avait pas encore été mis en français ${ }^{9}$ et c'est bien à cette tâche qu'Étienne de Laigue s'attela dans une période dont je pense qu'elle n'est pas antérieure à 1525 . En tout état de cause, l'édition traduite en français du corpus césarien parut pour la première fois à Paris en 1531, sortie des presses de Galiot du Pré pour Poncet le Preux. Avant d'entrer dans le détail de cette traduction, il convient de s'arrêter un peu sur la date probable de composition du texte. Étienne de Laigue semble avoir remis presque en même temps à Galiot du Pré les quatre textes qui composent l'intégralité de son œuvre conservée: le commentaire de Pline occupe à lui tout seul presque cinq cents feuillets, et même si l'on admet que la composition du Traicté des tortues et de l'Encomium a pu être relativement rapide, il fallait tout de même plusieurs mois pour traduire ceux des textes du corpus césarien qui n'existaient qu'en latin.

Il est bien entendu impossible de déterminer le temps nécessaire à la composition de ces quatre œuvres ; mais je peux plus sûrement tenter de leur donner un ordre et si possible un terminus a quo.

Le traité des tortues cite le commentaire de Pline; il faut donc admettre qu'il a été composé postérieurement. Le commentaire de Pline, de son côté, répond à la publication des Annotationes in Plinium de Beatus Rhenanus, parues en $1526^{10}$ : il n'a donc pu être rédigé qu'entre 1526 et 1530 . Il cite en outre le commentaire sur l'œuvre de Martianus Capella, dont on ne sait à peu près rien, mais qui pourrait être antérieur aux années 1526-1530. Tout se passe comme si Étienne de Laigue avait profité d'un moment de répit, entre la fin de la campagne d'Italie et le début de ses missions diplomatiques pour s'adonner à des travaux d'érudition classiques. On ne peut exclure que ce répit soit en liaison avec une immobilisation forcée en Espagne. L'absence de publication avant Pavie et après 1530 s'expliquerait alors assez simplement par l'intensité des activités militaires et diplomatiques du seigneur de Beauvais.

Il faut à présent en venir au corpus césarien, vu par Étienne de Laigue. En croisant les observations faites par Frédéric Duval dans son article sur Robert Gaguin et l'examen direct d'un certain nombre de ces éditions, il faut conclure que cette édition française du corpus césarien a rencontré un succès certain. La première

trouvera quelques indications sur les premières éditions italiennes du corpus césarien au début de l'étude suivante: Virginia Brown, The textual transmission of Caesar's civil war, Leyde, Brill, 1972.

${ }^{9}$ Il existait cependant des versions vernaculaires du corpus césarien dans d'autres langues. Je remarque tout particulièrement une édition espagnole immédiatement antérieure au travail d'Étienne de Laigue. Si le passage du diplomate français par la péninsule ibérique était avéré, la publication de l'ensemble de la traduction du corpus en 1529 aurait pu être l'élément déclencheur pour la mise en œuvre de la traduction d'Étienne de Laigue.

${ }^{10}$ Beatus Rhenanus, In C. Plinium. Repurgatur hoc libro non solum prafatio Pliniana a multis mendis et ipsi Naturalis historia libri infinitis locis castigantur, Bâle, 1526. 
édition parut à Paris en $1531^{11}$. Elle fut suivie, par ordre chronologique des éditions suivantes :

Paris, Jean Petit, $1537^{12}$

Paris, Arnould et Charles les Angeliers frères, $1539^{13}$

Paris, Jean Petit, $1539^{14}$

Paris, imprimé par P. Gaultier pour Jehan Barbé, $1545^{15}$

Paris, par Pierre Gaultier, 1546

Paris, pour Galiot du Pré, c. 1550

Paris, Guillaume Thiboust, 1555

Lyon, Jean de Tournes, 1555

Paris, Antoine Bonnemere, 1559

Paris, Jehan Ruelle, 1569

Paris, Nicolas Bonfons, 1577

Même s'il se trouve sans doute dans cette liste quelques omissions, il ressort clairement que le travail d'Étienne de Laigue n'a pas été jugé négligeable ${ }^{16}$. L'arrêt des rééditions dans les années 1576-1577 s'explique assez aisément par la publication de la seconde édition du corpus français de César à la Renaissance due, cette fois, au travail érudit de Blaise de Vigenère ${ }^{17}$.

Les éditions d'Étienne de Laigue se caractérisent par des variations très importantes du format. Si la première est un petit in-folio, les suivantes adoptent une taille plus petite selon un modèle qui pourrait être celui des éditions latines contemporaines. Ces rééditions ont d'autre part pour caractéristique d'être presque toutes posthumes. On peut avoir un doute sur l'édition de 1537 bien qu'il soit peu probable que, pris dans des missions diplomatiques continues, l'auteur ait eu le loisir de procéder à des révisions. En revanche, on a la preuve pour plusieurs de ses œuvres que d'autres ont pu intervenir, quelquefois d'une façon massive. Le commentaire de Pline, par exemple, a fait l'objet d'une réédition partielle en 1548. Il s'agissait alors d'isoler du texte général les pages concernant le début du livre XXX, relatif à la magie ${ }^{18}$. Associé à d'autres textes thématiquement proches, ce texte a

\footnotetext{
${ }^{11}$ Inventaire chronologique des éditions parisiennes $d u X V I^{e}$ siècle, t. 3 (1531-1535), Paris, $2004, n^{\circ} 68$, p. 68 . La notice précise que la deuxième partie qui concerne la Guerre des Gaules, suit l'édition de 1521.

${ }^{12}$ Inventaire chronologique des éditions parisiennes du XVI siècle, t. 5 (1536-1540), Paris, 2004, n 392, p. 156.

${ }^{13}$ Inventaire chronologique des éditions parisiennes du XVI ${ }^{e}$ siècle, t. 5 (1536-1540), Paris, 2004, n 1191, p. 353.

${ }^{14}$ Inventaire chronologique des éditions parisiennes $d u X V I^{e}$ siècle, t. 5 (1536-1540), Paris, 2004, n 1190 , p. 353.

${ }^{15}$ Imprimeurs et libraires parisiens du XVI siècle : ouvrage publié d'après les manuscrits de Philippe Renouard, Paris, 1979, t. 3, p. 37, n 40.

${ }^{16}$ Ma liste est simplement indicative. Elle ne vise pas l'exhaustivité.

${ }^{17}$ César, Jules, Les Commentaires de César, des Guerres de la Gaule mis en françois par Blaise de Vigenère, avec quelques annotations dessus, Paris, Nicolas Chesneau, 1576.

${ }^{18}$ In Caii Plinii Secundi Naturalis historia... 1 et 2 cap. libri XXX commentarius, naturalis quidem magia compendiariam rationem... totiusque natura cognitionem complectens.] Cui...
} 
donc été republié sans l'accord de son auteur. La situation est à peu près la même dans le cas du Traité des Tortues. L'édition de 1530 ne mentionne que les quatre objets explicitement désignés dans le titre. Mais une édition postérieure, datée de 1546, ajoute au texte initial deux chapitres portant l'un sur les champignons et l'autre sur les courges. Comme on voit, en dépit des jugements souvent négatifs portés sur le travail du seigneur de Beauvais, il semble qu'aucune de ses œuvres n'ait laissé le monde savant indifférent.

En outre, la constitution du corpus français de César n'était pas une œuvre absolument originale : elle s'inscrit très clairement dans la continuité des traductions du De Bello gallico et il devient, de ce fait, indispensable de mesurer les ruptures et les continuités de cette tradition. Il faut donc entrer dans le détail du corpus constitué pour y voir plus clair ${ }^{19}$.

La page de titre de l'édition de 1531 annonce un programme complet:

Les Commentaires de Jules Cesar : de la Guerre civile, de la guerre alexandrine, de la guerre d'affrique, de la guerre despaigne, translatez par noble homme Estienne Delaigue dict Beauvoys. Des batailles par feu de bonne memoire Robert Gaguin général de l'ordre de la saincte Trinité. Avec les portraicts et descriptions des lieux fortz, pontz, machines et aultres choses dont est faict mention es presens commentaires. Ensemble les noms des lieux, villes et peuples du pays de Gaule. Avec privilège du Roy nostre Sire. On les vend a Paris en la grand rue Sainct Jacques devant les Maturins en la boutique de Poncet le Preux, 1531.

Pièces liminaires :

[1] Extrait de privilège de François $\mathrm{I}^{\mathrm{er}}$; Table de ce present livre des commentaires de Jules $\operatorname{cesar}^{20}$;

[2] Lettre dédicatoire à Philippe Chabot du Brion

[3] Les portraictz des choses memorables des commentaires de Cesar. L'édition de 1531 présente en raison de son format des cartes et des plans de grande taille ${ }^{21}$.

Les noms des lieux, villes et peuples du pays de Gaule, dont mention est faite aux commentaires de Jules Cesar.

À la fin du volume :

«Cy finist la translation des commentaires de Jules Cesar sur le faict de la conqueste du pays de Gaule faicte et mise en francoys par frere Robert gaguin docteur en

adjecta est de fascinationibus disputatio elegans... Item de incantatione et adjuratione... epistola incerti authoris, una cum Joannis Tritemii,... in libros suos Steganographia epistola apologetica, etc., quibus difficultates pliniana, prasertim magica, omnes explicantur, Würzburg, J. Mylius, 1548.

${ }^{19}$ Sauf indication contraire, je cite l'édition de 1531. L'exemplaire consulté est celui de la bibliothèque nationale de France, conservé sous la cote J 999 (RES).

${ }^{20}$ L'édition de 1531 donne le corpus césarien dans l'ordre suivant : De Bello ciuili; De Bello alexandrino; De Bello africano; De Bello gallico.

${ }^{21}$ Le programme iconographique est profondément différent de celui mis en œuvre dans les éditions de la traduction de Gaguin pour la guerre des Gaules. 
decret et general ministre de lordre des freres de saincte trinite et redemption des prisonniers chrestiens »;

[4] Poème post-liminaire ; corrections en limpression ; colophon :

«Ce present oeuvre des commentaires de Jules Cesar, tant des guerres civiles que Gaule, ont este achevez dimprimer en la noble ville et cite de Paris le XVIII jour du moys de may, lan mil cinq cens trente et ung, par maistre Pierre Vidoue imprimeur, et libraire jure, Pour honnestes personnes Poncet le Preux et Galiot du Pre libraires jurez de l'universite dudict lieu. M.D.XXXI.

Le texte de la Guerre des Gaules est précédé du prologue de Robert Gaguin. La comparaison du texte de l'édition de Gaguin de 1531 avec des éditions antérieures conduit à penser qu'Étienne de Laigue n'a pas modifié le texte français. Les seules variations, et elles existent, concernent les conventions orthographiques ${ }^{22}$.

Il convient donc, à présent de revenir sur l'ensemble de pièces liminaires qui entourent le corpus césarien, en commençant par la longue série des «protraicts (sic)». Il s'agit d'un ensemble de cinq planches et deux cartes décrivant successivement divers lieux du pays de Gaule ${ }^{23}$. Ces planches s'inscrivent si naturellement dans le propos du commentateur d'éclairer des points essentiels des campagnes de César qu'on a bien envie de savoir gré à Étienne de Laigue d'avoir offert au lecteur un tel instrument d'approche de la réalité césarienne.

Or, on aurait partiellement tort.

On peut savoir gré à l'auteur de ces planches d'avoir précisé par le dessin les réalités de César, mais cet auteur n'est pas Étienne de Laigue. Ces planches constituent l'une des pièces liminaires classiques du texte de César depuis l'édition aldine publiée en 1513. Elles sont en réalité l'œuvre de Fra Giocondo auquel on attribue aussi la responsabilité philologique de l'édition vénitienne. Cet ensemble de bois est repris dans les éditions postérieures latines du corpus, avant et après 1531 . La table des commentaires, l'index des villes ne me semblent pas non plus relever du travail d'Étienne de Laigue qui s'est contenté, en cette matière, de traduire ce qui préexistait dans les éditions latines de César. Son intervention réside plutôt dans le choix d'une disposition particulière des éléments d'accompagnement plutôt que dans l'invention d'un nouveau dispositif iconographique.

Ainsi, pour la Guerre des Gaules, Étienne de Laigue n'est pas responsable de la traduction, pas plus que des planches : doit-on pour autant conclure qu'il n'a rien

\footnotetext{
${ }^{22}$ Je renvoie sur ce point à ce qu'en écrivait récemment Frédéric Duval dans cette même revue.

${ }^{23}$ «Le protraict et description du pont lequel Cesar fist sur le Rhin duquel il est fait mention au quatriesme livre de commentaire de Jules Cesar des batailles de Gaule. Le protraict et description de la forteresse de Avaricum dont il est faict mention au septiesme livre des commentaires de Jules Cesar des batailles de Gaule. Le protraict et description de la forteresse de Alexia de laquelle il est fait mention au septiesme livre des commentaires de Jules Cesar des batailles de Gaule. Le protraict de la forteresse et ville de Uxellodunum dont il est faict mention au huytiesme livre des commentaires des batailles de Gaule faict par Hirtius. Le protraict et description de la ville de Marseille de laquelle il faut mention au second livre des commentaires de Jules Cesar de la guerre civile. Le protraict du pays despaigne».
} 
fait et qu'il s'est contenté de récupérer le travail de Robert Gaguin ? Il faut sans doute aller un peu plus loin dans l'analyse.

Robert Bossuat, dans un article assez ancien, affirmait que l'on devait à Étienne de Laigue la division en chapitres du texte français de la Guerre des Gaules $^{24}$. Il semble que ce soit encore trop prêter d'honneur à notre commentateur. S'il est vrai que l'édition Vérard ne comporte aucune division, la division en chapitres préexiste dans l'édition des Euvres et briefves expositions de Jules Cesar sur le faict des batailles de Gaule de la traduction de Robert Gaguin, imprimée à Paris par la veuve de Michel Le Noir. Comme les éditions connues sous la responsabilité de la veuve de Michel Le Noir datent des années 1521, il faut donc conclure à l'antériorité de cette édition sur la publication de la traduction d'Étienne de Laigue. Pour dire la vérité, cette division est aussi, ipso facto, postérieure à la mort de Gaguin. Sauf à considérer que les Euvres et briefves expositions ont été publiées par Étienne de Laigue, et l'on n'a aucune raison de le faire, il faut donc renvoyer à une autre responsabilité cette division pourtant bien commode. Au reste, le sens retenu par les titres affectés à chacun des chapitres va bien dans le sens idéologique de la traduction de Robert Gaguin: il s'agit toujours de magnifier le grand capitaine en soulignant la perspicacité de ses décisions militaires ou son sens de la clémence: ainsi au livre III, chapitres 3 et 4, trouve-t-on successivement les deux titres suivants: "Comment ceulx de Vannes feirent leurs preparatifs, et manderent secours en plusieurs lieux » et «comment Cesar envoya plusieurs de ses lieutenenz en plusieurs pays de Gaule pour empescher les secours de ceulx de Vannes et comment Cesar par son industrie couppa les cordaiges des navires de ceulx de Vannes, et coment bien peu se sauverent dont la guerre fina. Et apres se rendirent a Cesar qui fist occire tous les conseilliers et vendit les autres au plus offrant pour cause quilz avoient emprisonne et retenu les ambassadeurs et ses commiz et lieutenenz ».

Pour l'édification morale, on se reportera par exemple au titre du chapitre XVIII du livre V : «Comment deux bons et vaillants capitaines Romains qui avoient eu continuel debat ensemble pour la preeminence de dominer, secoururent l'ung l'autre à ung tres grand besoing contre Ambiorix ».

Il ne faudrait cependant pas conclure de ces remarques qu'Étienne de Laigue n'a rien fait pour la Guerre des Gaules et que son intervention s'est limitée à reprendre un appareil de notes déjà constitué ailleurs. Son apport est sensible dans les notes marginales assez nombreuses de l'édition. On ne s'étonnera pas de constater l'absence de notes proprement philologiques: le problème ne se posait pas en ces termes. En revanche, les notes sont majoritairement explicatives. Elles ont pour but de situer les peuples mentionnés par César et d'établir plus nettement le lien avec les villes connues de ses contemporains. La logique de cette série de notes est donc exactement parallèle à celle qui prévalait dans l'esprit de Giovanni

${ }^{24}$ Robert Bossuat, "Traductions françaises des commentaires de César à la fin du $\mathrm{XV}^{\mathrm{e}}$ siècle », BHR, t. IV, 1944, p. 381-382: «La disposition des matières, les innovations introduites par l'éditeur révèlent un souci très net de vulgarisation», et, p. 382 : «Le texte de Gaguin est exactement celui des anciennes éditions de Vérard et contient la dédicace à Charles VIII. Mais il est divisé en chapitres ayant chacun leur rubrique particulière et pourvu de courts sommaires dans les marges extérieures». 
Giocondo, lorsqu'il imagina les planches des trois villes d'Avaricum, Alesia et Uxellodunum. D'autres notes, tout en restant explicatives, établissent sur le terrain des idées le parallèle entre César et la France de François I ${ }^{\mathrm{er}}$. L'un des meilleurs exemples vient du livre IV de la Guerre des Gaules, dans un passage où César évoque l'autorité des druides. Je cite le texte de César d'abord, la note d'Étienne de Laigue ensuite :

et se il y aulcun petit ou grand, qui ne veuille obeyr à leurs ordonnances, ilz luy defendent d'estre present à leurs sacrifices : laquelle chose est reputée entre eulx vie de moult grieve peine. Car ceulx qui sont aussi banniz et privez de sacrifices, sont tenuz et mys en nombre de cruelz et mauvais garsons : et chascun les fuit, et chascun les deboute de son hostel, et se gardent de parler à eulx». Voici maintenant la note d'Étienne de Laigue: «Nota : ceste peine est comparée à l'excommunication que faict aujourduy l'esglise contre les delinquentz .

Ces parallélismes de civilisation s'étendent à de menus détails aussi bien qu'à des vérités très générales : «Les Francoys sont fort donnez à devotion » ou encore «les fenerailles des Francoys sont sumptueux». De telles notes s'inscrivent dans une double perspective: d'une part, elles font assez nettement écho à la dimension ethnologique du texte latin de César, sensible lui aussi, aux particularismes des civilisations; d'autre part, par le renforcement de la dimension exemplaire de la figure de César, elles permettent de réactualiser en 1531 le projet idéologique de Robert Gaguin.

Il faut à présent revenir sur une des autres pièces liminaires de l'édition de 1531 , la lettre de dédicace à l'amiral Philippe Chabot du Brion. Ce texte comporte, comme on pouvait s'y attendre, les thèmes essentiels caractéristiques du genre : louange du dédicataire, reconnaissance d'une certaine dette à son égard, mise en exergue de la convergence entre les préoccupations du dédicataire et la thématique du texte dédié. On retiendra par exemple les phrases suivantes : «considérant ces choses, me suis ingeré te offrir ce present, c'est à dire ceste traduction des commentaires de Jules Cesar de la guerre civile pensant que la lecture diceux sera non seulement delectable, ains commode et fructueuse à plusieurs, principalement pour animer la noblesse francoyse à magnanimité et faict militaire, attendu l'industrie, scavoir, astuce que Cesar demontre en ceste œuvre». Du côté de l'humilité, du traducteur on retiendra ces deux tournures qui ouvrent et clôturent le texte de la dédicace : «La singuliere et commendable modestie, prudence et magnanimité m'ont meu, te dedier cest œuvre, Illustre seigneur, je scay pour vray ta debonnaire nature, civilite, et clemence n'estre moindre que celle du tres opulant le magnanime Roy de Perse Xerxes, auquel allant par pays un homme affligé de extreme indigence presenta ses deux plaines mains d'eau naiant autre chose que il peust presenter à la majesté royale, ce neantmoins cestuy present le Roy receut de telle benivolence, que s'il eust esté plus magnifique et precieux »; et à la fin de la lettre : "et si ce que te offre à present est de petite estimation ton bon plaisir sera excuser la mienne petite portée et facultée, et supporter mon default». On remarquera au passage que le discours d'humilité sur soi-même, celui qui met en évidence la faiblesse de l'auteur est une constante étonnante chez Étienne de Laigue ; elle apparaît tout particulièrement à la 
fin de l'épitre dédicatoire du Singulier traictée $e^{25}$, dans la tournure «petit donne qui petit a, Chascung n'a pas Beaulvais ». Il n'est pas impossible que ce thème récurrent dissimule un jeu sur l'identité réelle de l'auteur du texte: il existait bien un autre Beauvais, beaucoup plus important que sa terre du Berry ${ }^{26}$; il n'est pas impossible non plus qu'en avançant l'humilité de sa situation l'auteur ne se cache derrière une de ses possessions mineures et qu'il soit par ailleurs détenteur de terres beaucoup plus importantes.

Si l'on tente un résumé de la situation, voici les points saillants de l'apport d'Étienne de Laigue sur le texte de la Guerre des Gaules: un grand capitaine qui a bien servi François $\mathrm{I}^{\mathrm{er}}$ dans des temps difficiles après Pavie traduit César. Pour la singularité de son travail et sans doute aussi parce qu'il se prépare à l'envoyer servir sa politique étrangère partout en Europe, François $\mathrm{I}^{\mathrm{er}}$ lui accorde un privilège. C'est donc bien à la lumière non des campagnes de Charles VIII mais de celles de François $\mathrm{I}^{\mathrm{er}} \mathrm{qu}$ 'il faut lire la cohérence de cette publication.

Il faut à présent en venir au reste du corpus de César sur lequel l'intervention d'Étienne de Laigue est encore plus nette. Les remarques faites sur les aménagements apportés au texte de Gaguin, la cohérence des remarques marginales restent globablement valables pour la suite du corpus. La typologie des notes d'Étienne de Laigue s'avère cependant légèrement plus variée dans la partie du corpus dont il est plus directement responsable. Plus que dans la Guerre des Gaules, Étienne de Laigue procède régulièrement au rapprochement des sources historiques : Lucain et Tite Live sont alors régulièrement convoqués, ainsi que la correspondance de Cicéron. Du côté des modernes, la seule référence notable concerne Guillaume Budé, dont le De Asse est ainsi régulièrement cité. Il se cite également, au moins deux fois : d'abord à propos de la description de Marseille et ensuite à propos de la forme du delta du Nil et de la position de la ville d'Alexandrie. Le seigneur de Beauvais renvoie dans les deux cas à son commentaire sur Pline, ce qui confirme l'antériorité de ce texte sur la traduction de César et renvoie donc la date de composition de la traduction dans les années 1526-1530.

Quand elles ne sont pas d'érudition historique, les notes d'Étienne de Laigue se font assez régulièrement lexicales : à propos de l'expression agere cum populo, le

${ }^{25}$ « Je ne te pense de si rude, et agreste Minerve, que ne conjecturez es petites choses consister et estre grandissime et memorable dignité. Ce assez témoignent les petites pierres précieuses, dyamans, saphirs, rubys, esmeraudes, escarboucles, petites à veoir, grandes à calculer et considérer leur occulte efficace, opération admirable, et ineffable. Que dys tu des abeillez, des formys, des yraignez, desquelz l'industrie, l'excellence, la providence, l'artificieuse opération, la sédulité incomparable, l'active raison excède toutes bestes et leur action. Je ces choses de attentif intellect examinant, me suys ingeré ce petit, exile, non copieulx traicté te donner, simple et petit est, ce neautmoins de voloir bon à toy exibe, petit donne qui petit a, Chascung na pas Beaulvais».

${ }^{26}$ La seigneurie de Beauvais reste mal identifiée. Un opuscule anonyme, qui est peut être de la main de Louis de Laigue, l'associe à une commanderie située près de Buzançais, également mentionnée dans les descriptions archéologiques du Bas Berry. V. Notice historique sur Notre-Dame de Beauvais, Paris, 1856 et Henri Vaillant, Ingrandes et les confins du BasBerry, Châteauroux, 1904. Cependant, l'inventaire des archives de l'Indre, établi par l'archiviste Eugène Hubert fait apparaître plusieurs autres lieux du même nom. 
commentateur ajoute la remarque suivante: Agere cum populo est rogare populum quid suffragio suo vel jubeat vel vetet. Unde rogatio pro lege dicta. Auctores Festus Gellius et Macrobius. Cette orientation grammaticale du commentaire dans les marges explique le recours assez constant à Aulu Gelle, Festus ou Macrobe.

Indépendamment des notes que je qualifierais d'explicatives, il existe aussi dans le texte des notes en français qui répondent davantage à la fonction d'articulation du sens, d'entrée de table que de commentaires à proprement parler. Ailleurs, une note précisant «Excuse que fait César se voulant purger n'estre cause de la guerre civile» serait assortie d'une manchette : mais il n'y a pas de manchette dans l'édition de 1531 et aucun index n'exploite ces indications.

Dans cet ensemble, les notes philologiques sont particulièrement rares et c'est sur elles cependant qu'il faut se pencher pour tenter de comprendre le problème du traitement des sources textuelles dans le commentaire. Avant de regarder les pauvres détails donnés dans les marges du texte, il convient d'attirer l'attention du lecteur moderne sur plusieurs difficultés. La première tient dans l'appréciation des écarts entre la version française et la version latine. L'intervention du traducteur modifie fréquemment les rapports entre la version latine du texte et la version qu'on attendrait aujourd'hui en français. Une autre difficulté tient à la diversité des sources possibles. Pour faire une traduction, un humaniste peut couramment utiliser une édition latine imprimée relativement récente. Le recours à d'autres traditions textuelles dans la langue d'origine est toujours possible, mais il n'est pas nécessairement attesté. Autrement dit, on peut corriger une traduction du latin vers le français en se reportant au texte latin d'origine, en consultant d'autres éditions imprimées latines qui offrent des variantes textuelles, en faisant une collation avec des manuscrits ou en confrontant avec d'autres traductions françaises. On peut même corriger en se reportant à la connaissance profonde que l'on a des réalités romaines décrites dans le texte. Le choix dépend à la fois du degré de sensibilité philologique du traducteur et du matériel textuel disponible pour une œuvre donnée.

Pour le corpus de César la difficulté est la suivante : la tradition manuscrite de César et du pseudo-César est particulièrement abondante. L'éditrice du Bellum hispanicum note dans son introduction 162 recentiores $^{27}$. Un bref coup d'œil au fichier des manuscrits latins de l'Institut de recherche et d'histoire des textes montre que l'on peut sans doute en compter davantage, notamment si on prend en considération l'intégralité du corpus césarien. Le dernier élément à prendre en considération tient au temps dont Étienne de Laigue disposait pour réaliser son travail. Même en l'imaginant isolé, immobilisé aux côtés des enfants de France, il n'a pas pu se livrer à de longues collations en plus de la traduction, du commentaire de Pline et de la rédaction des deux opuscules. Dès lors, il faut s'orienter vers une interprétation économique des sources textuelles.

Les variantes textuelles sont exceptionnellement peu nombreuses et deux d'entre elles seulement sont exploitables. Deux autres ne sont pas, dans l'état actuel

\footnotetext{
${ }^{27}$ Pseudo-César, Guerre d'Espagne, texte établi et traduit par Nicole Diouron, Paris, Les Belles Lettres, 1999, p. CIV-CV. L'auteur utilise alors la liste de manuscrits établie par Virginia Brown dans The textual transmission of Cosar's civil war, Leyde, Brill, 1972 ; et Wolfang Hering, Die Recensio der Casarhanchriften, Berlin, 1963.
} 
de mes connaissances, directement interprétables. Les deux variantes utiles proviennent du Bellum alexandrinum.

Au début du chapitre XII consacré à Vatinius et à la bataille navale de Tauris, le passage problématique est celui où l'auteur fait le bilan des gains de César au terme de cette bataille. Étienne de Laigue note alors dans la marge : «Certains lisent dicrotas et non dyerotas. Il s'agit de navires dotés d'un double gouvernail qui peuvent être gouvernés depuis la proue ou depuis la poupe ${ }^{28} »$. L'examen de l'editio princeps de Giovanni Andrea Bussi permet de faire avancer les choses : c'est bien la variante dyerotas qui apparaît et c'est bien une leçon fautive. Dyerotas est encore la leçon de l'édition aldine de 1513, de l'édition parisienne de 1528. En revanche, l'édition bâloise de la même année atteste dicrotas, de même que la correction manuscrite de Piero Vettori portée dans les marges de l'exemplaire de l'édition princeps aujourd'hui conservé à la Bibliothèque nationale de France ${ }^{29}$. Il n'était donc pas nécessaire de retourner à une leçon hypothétiquement correcte dans la tradition manuscrite pour corriger le texte sur ce point, et d'autant moins que la note portée dans l'édition moderne de la Collection des Universités de France qui signale ce mot comme un hapax n'est pas tout à fait juste. Le mot apparaît également dans les lettres à Atticus et l'on se rappelle que le seigneur de Beauvais citait explicitement ce texte dans l'épitre dédicatoire. On ne peut donc pas savoir si Étienne de Laigue a eu entre les mains l'édition bâloise de 1528 du corpus latin de César, mais il a pu corriger la leçon sur la seule foi d'une version non fautive des lettres à Atticus.

La seconde variante exploitable intervient à l'extrême fin du texte. Dans l'affrontement entre Cassius et Marcellus, il s'agit de savoir précisément si Marcellus se cantonne dans ses propriétés (pradium) ou dans un camp retranché (prasidium). Étienne de Laigue précise alors : «dans certains textes (codicibus) on lit prcediis. Je préfère lire pour ma part prasidiis ainsi que le sens l'exige ${ }^{30}$. Sur ce point encore, c'est sur la tradition imprimée qu'Étienne de Laigue nous renseigne : la version fautive prcediis apparaît dans l'édition aldine de 1513, dans l'édition parisienne de 1528, dans l'édition princeps, et dans une majorité d'éditions anciennes. L'édition bâloise donne bien prasidiis, mais la tournure de la phrase d'Étienne de Laigue invite à voir dans cette correction la logique du sens plutôt que celle de la source textuelle. C'est bien la leçon prasidiis qui est aujourd'hui couramment retenue.

En ajoutant les arguments les uns aux autres, je peux presque arriver à la conclusion qu'Étienne de Laigue n'avait pas de manuscrit de César sous la main, alors qu'il disposait d'au moins un manuscrit de l'Histoire naturelle de Pline. On en saurait évidemment davantage si l'on pouvait déterminer avec précision où se trouvait ce curieux personnage entre le désastre de Pavie et la publication du texte. On conviendra néanmoins que dans les deux cas cités, il ne s'est pas trop mal tiré d'affaire.

\footnotetext{
${ }^{28}$ F. 59v: alii non dyerotas sed dicrotas legunt. Sunt enim nauesque duobus gubernaculis pariter a prora reguntur et puppi.

${ }^{29}$ Casaris Commentarii. Item Auli Hirtii, aut Oppii, libri de bello Alexandrino, de bello Africo et de bello Hispano. Edente Joanne Andrea, Rome, 1469. BnF [RES- J- 256 ]

${ }^{30}$ Bellum Alexandrinum, chap. 63.
} 
Le dernier point sur lequel il convient d'attirer l'attention du lecteur est relatif au style de la traduction. Il n'entre pas dans mes intentions de proposer ici au lecteur une analyse complète du style d'Étienne de Laigue. Mon avis, subjectif, est que sa plume manque de grâce aérienne. Même quand la syntaxe est exactement comprise, elle peut aboutir à des versions que la langue française n'admet qu'avec peine: qu'on en juge à l'aune de cette version du début de la Guerre Civile: "Or plusieurs avoient esté de plus civile et humaine opinion comme premierement Marcus Marcellus disant ces propoz que on ne devoit faire consultation, ne mettre ceste chose pour deliberer vers le senat que auparavant par toute l'italie ne fust faict amatz de gens de guerre, et iceulx enroullez afin que à leur aide seulement et en liberté le senat peust decreter ce qu'il vouldroit, comme aussi Marcus Calidius... » la phrase n'est pas terminée. Plus généralement, à côté de belles trouvailles stylistiques, on trouve aussi de nombreuses lourdeurs qui obligent à reconstruire la syntaxe de la phrase en français, comme il est naturel de le faire en latin.

Sur le plan des figures, la plus fréquente et la plus remarquable tient dans le redoublement, figure que j'interprète à tort ou à raison comme une continuation de la tradition médiévale ${ }^{31}$ : dans les deux exemples suivants le latin ne nécessitait nullement un redoublement : «Semblable propos fut confirmé par Scipion disant que Pompée avoit deliberé ne faillir a la chose publicque, moyennant que le Senat voulust adherer à lui, et estre de son avis». ou encore : « et est la puissance et liberté de decreter à plusieurs tollie et ostee ». Quelquefois, le redoublement peut prendre des proportions beaucoup plus graves :

Et pour ces raisons et causes les choses et négoces sont faictes soudainement et sans maturité et premedité conseil et sans donner loisir ne espace de temps au parens et voisins de Cesar, ne aux tribuns du populaire de l'advertir.

Le même passage est ainsi traduit par Nisard :

Par ces motifs, tout se décide à la hâte et en tumulte ; on ne donne pas le temps aux parents de César de l'avertir

Le texte latin donnait quant à lui laconiquement :

His de causis aguntur omnia raptim atque turbate. Nec docendi Casaris propinquis eius spatium datur nec tribunis plebis sui periculi deprecandi.

Je ne sais pas s'il faut lire dans ce trait stylistique appuyé la volonté de dramatiser les conflits qui mèneront à la guerre civile. Il est clair toutefois, d'une part que ce trait stylistique établit nettement une continuité avec un mode d'expression médiéval, et, de l'autre, que les redoublements systématiques constituent un curieux traitement de la brièveté de César. Ce trait stylistique est d'ailleurs moins fréquent dans les œuvres originales d'Étienne de Laigue sans toutefois être totalement absent. Les autres particularités du style de César,

${ }^{31}$ Sur ce point, je renvoie à Frédéric Duval, art.cit, qui fait le point sur la question du redoublement synonymique à propos de la traduction Gaguin. Il semble qu'Étienne de Laigue ait quelquefois moins de scrupules que le traducteur de la Guerre des Gaules. 
notamment l'utilisation du style indirect libre, sont mieux respectées: dans ce cas précis, Étienne de Laigue passe alors au style direct.

Il reste pour conclure à tenter d'évaluer l'importance des traductions d'Étienne de Laigue dans la tradition de César. Son apport le plus important réside sans aucun doute dans la volonté de structurer en français un corpus jusque là diffusé en latin. Pour le Bellum ciuile, il s'agit de l'une des toutes premières traductions. L'appareil de notes installé sur tous les textes du corpus participe de la volonté d'explicitation du texte, voire de vulgarisation. Il est même permis de se demander si la réduction du format dans les éditions postérieures à 1531 ne pourrait pas être l'indice d'une utilisation scolaire.

Au-delà de 1576, le jugement porté sur le travail du seigneur de Beauvais ne bénéficiera plus de la moindre bienveillance. Au XVIII ${ }^{\mathrm{e}}$ siècle, $\mathrm{M}$. de Wailly faisait dans la préface à sa propre traduction le point sur les travaux antérieurs et voilà ce qu'il écrivait ${ }^{32}$ : "Nous avons en notre langue plusieurs traductions de ces commentaires : l'une est de Robert Gaguin qui vivait en 1500, la seconde est de Blaise de Vigenère dans le seizième siècle. Nicolas Perrot d'Ablancourt en donna une troisième vers le milieu du XVII ${ }^{\mathrm{e}}$ siècle. Les deux premières sont tout à fait oubliées. Celle d'Ablancourt a eu autrefois beaucoup de vogue...». On peut arrêter là le pénible rappel d'un réquisitoire dont Perrot d'Ablancourt est la victime. Comme on le voit, Étienne de Laigue n'est même pas mentionné dans la liste des traducteurs. C'est dire si son travail, qui a cependant au moins permis à la traduction de Robert Gaguin de perdurer au XVI ${ }^{\mathrm{e}}$ siècle, est définitivement oublié. Il reste que ce travail témoigne amplement de la réception du corpus de César dans un milieu qui avait toutes les raisons d'être sensible aux qualités d'homme de guerre, aux talents diplomatiques du dictateur romain. Il reste aussi que le corpus rassemblé par Étienne de Laigue permet au projet politique qui sous-tend la traduction Gaguin de trouver continuité et aboutissement dans la période suivante du règne de François $\mathrm{I}^{\mathrm{er}}$. Cela seul sauverait Étienne de Laigue, s'il en était besoin, l'homme qui entre deux chevauchées aux côtés de son roi trouvait encore le temps de traduire César, de commenter Pline et de s'interroger gravement sur les tortues, les escargots, les grenouilles et les choux.

Marie-Élisabeth Boutroue

${ }^{32}$ Jules César, Les Commentaires de César en latin et en françois revue par M. de Wailly, Paris, Barbou, 1775. Comme les précédentes, cette traduction de l'œuvre de César a connu plusieurs rééditions. 